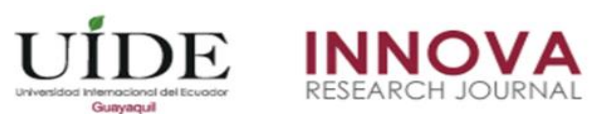

INNOVA Research Journal, ISSN 2477-9024

(Octubre, 2018). Vol. 3, No.10.1 pp. 55-67

DOI: https://doi.org/10.33890/innova.v3.n10.1.2018.785

URL: http://revistas.uide.edu.ec/index.php/innova/index

Correo: innova@uide.edu.ec

\title{
Las certificaciones ambientales ecuatorianas en la competitividad de las empresas
}

\section{Ecuadorian environmental certifications in the competitiveness of companies}

\author{
Andreina Inés González Ordóñez \\ Universidad Metropolitana, Ecuador
}

Autor para correspondencia: aigonzalez@umet.edu.ec andreinagonzalez09@gmail.com Fecha de recepción: 23 de agosto de 2018 - Fecha de aceptación: 01 octubre de 2018

Resumen: Las certificaciones ambientales otorgan beneficios ecológicos y empresariales debido a que permiten a las empresas desarrollar procesos más limpios que contribuyen en la mejora ambiental y abren paso a mercados competitivos. En esta investigación se analizan las certificaciones ambientales ecuatorianas y su contribución en la competitividad de las empresas. El tipo de investigación es bibliográfica con un diseño documental a partir de artículos de revistas, trabajos de grado y literatura relacionada con el tema estudiado, empleando como técnicas el análisis de contenido y contrastación de bibliografía. Los resultados obtenidos denotan que las certificaciones ambientales que otorga el Ministerio del Ambiente del Ecuador son la certificación punto verde y la certificación carbono neutro, encontrándose que un gran porcentaje de las empresas ecuatorianas todavía no cuentan con estas certificaciones, siendo una de las causas que las mismas todavía no cuentan con las capacidades para aplicar a estas certificaciones. Se concluye que las certificaciones ambientales contribuyen a que las empresas mejoren su competitividad a través de la rentabilidad económica y el crecimiento de las ventas, además de una mejor imagen corporativa, un mejor desempeño legal, un adecuado manejo de los recursos, la reducción en la generación de residuos y la atracción de nuevos clientes.

Palabras Claves: certificación ambiental; gestión ambiental; competitividad; empresas

\begin{abstract}
Environmental certifications provide ecological and business benefits because they allow companies to develop cleaner processes that contribute to environmental improvement and open the way to competitive markets. This research analyzes Ecuadorian environmental certifications and their contribution to the competitiveness of companies. The type of research is bibliographic with a documentary design based on journal articles, degree works and literature related to the subject studied, using as techniques the content analysis and bibliography comparison. The results obtained show that the environmental certifications awarded by the Ministry of Environment of Ecuador are the green point certification and the neutral carbon certification, finding that a large percentage of the Ecuadorian companies still do not have these certifications, being one of the causes that the They do not yet have the skills to apply for these certifications. It is concluded that environmental certifications contribute to companies improving their competitiveness through economic profitability and sales growth, as well as a better corporate image, a better legal performance, an adequate management of resources, the reduction in generation of waste and the attraction of new customers.
\end{abstract}

Key Words: environmental certification; environmental management; competitiveness; companies 


\section{Introducción}

Las certificaciones ambientales parten de la necesidad de las empresas de implementar estrategias para cumplir con procesos más limpios que les permita mitigar los impactos ambientales que generan y cumplir con la normativa ambiental. Poseer una certificación ambiental también le facilita a la empresa el ingreso a mercados competitivos, incrementa la rentabilidad de las mismas y les permite ser más competitivas. Por ello, para obtener estas certificaciones las empresas deben desarrollar y aplicar prácticas ambientales y producir cambios en sus procesos, de tal manera que no generen problemas ambientales y se adecuen a lo establecido en la normativa ambiental.

Las prácticas ambientales pueden abarcar reducción del consumo de agua y electricidad, gestión de desechos sólidos peligrosos y no peligrosos, reciclaje de materiales, uso de energías alternativas, eficiencia energética, entre otros.

La implementación de las prácticas ambientales y el desarrollo de procesos productivos más limpios en las empresas, además de permitir obtener la certificación ambiental, también contribuyen a mejorar su competitividad y al ingreso de las empresas a nuevos mercados o a los llamados mercados verdes.

En este orden de ideas, Rodríguez Becerra \& Van Hoof (2004) expresan que la gestión ambiental preventiva tiene como objetivo disminuir o evitar la contaminación y, al mismo tiempo, aumentar la productividad de la empresa, así como la calidad de los bienes y servicios producidos. Es un enfoque centrado en el mejoramiento continuo de los procesos productivos y productos, constituyéndose en una de las puertas de entrada para que los empresarios Pymes ingresen en los denominados mercados verdes, o una oportunidad para emprender nuevos negocios con alta competitividad. En este sentido, gestión ambiental y competitividad es un asunto que adquiere cada vez mayor significado si se toma en consideración el proceso de creciente internacionalización de los mercados y las nuevas exigencias de cumplimiento de la normatividad ambiental que conlleva.

El objetivo de la investigación fue analizar las certificaciones ambientales ecuatorianas y su contribución en la competitividad de las empresas. Para ello se estudió la base legal que le sirve de sustento, se identificaron los tipos de certificaciones ambientales que se otorgan en el país, se determinaron cuáles son las prácticas ambientales que han implementado las empresas para hacerse acreedoras de las certificaciones y por último se explica la contribución que han tenido las mismas en la competitividad de las empresas.

\section{Métodos}

El enfoque empleado en la investigación es el enfoque cuantitativo. El método empleado es el deductivo y su desarrollo fue a nivel exploratorio. El tipo de investigación es bibliográfica con un diseño documental a partir de artículos de revistas, trabajos de grado y literatura relacionada con el tema estudiando, empleando como técnicas el análisis de contenido y contrastación de bibliografía. 


\section{Resultados}

\section{Bases legales de las certificaciones ambientales ecuatorianas}

\section{Constitución de la República Ecuador}

La Constitución del Ecuador reconoce en el artículo 14 el "derecho de la población a vivir en un ambiente sano y ecológicamente equilibrado, que garantice la sostenibilidad y el buen vivir, sumak kawsay". Asimismo, el artículo 15 establece que el "estado promoverá en el sector público y privado, el uso de tecnologías ambientalmente limpias y de energías alternativas no contaminantes y de bajo impacto".

Por su parte, el artículo 66, numeral 27, "reconoce y garantiza a las personas el derecho a vivir en un ambiente sano ecológicamente equilibrado, libre de contaminación y en armonía con la naturaleza". El artículo 71 dispone que el "Estado incentivará a las personas naturales y jurídicas, y a los colectivos, para que protejan la naturaleza, y promoverá el respeto a todos los elementos que forman un ecosistema".

En este sentido, el artículo 278 señala que "para la consecución del buen vivir, a las personas y a las colectividades, y sus diversas formas organizativas, les corresponde producir, intercambiar y consumir bienes y servicios con responsabilidad social y ambiental" (numeral 2). En el artículo 395, numeral 2, se establece que "las políticas de gestión ambiental se aplicarán de manera transversal y serán de obligatorio cumplimiento por parte del Estado en todos sus niveles y por todas las personas naturales o jurídicas en el territorio nacional”.

En el artículo 408 se dispone que el "Estado garantizará que los mecanismos de producción, consumo y uso de los recursos naturales y la energía preserven y recuperen los ciclos naturales y permitan condiciones de vida con dignidad". El artículo 413 declara que "Estado promoverá la eficiencia energética, el desarrollo y uso de prácticas y tecnologías ambientalmente limpias y sanas, así como de energías renovables, diversificadas, de bajo impacto y que no pongan en riesgo la soberanía alimentaria, el equilibrio ecológico de los ecosistemas ni el derecho al agua".

\section{Código Orgánico Ambiental}

El Código establece en su artículo 5 el "derecho de la población a vivir en un ambiente sano. El derecho a vivir en un ambiente sano y ecológicamente equilibrado comprende: el desarrollo y uso de prácticas y tecnologías ambientalmente limpias y sanas, así como de energías alternativas no contaminantes, renovables, diversificadas y de bajo impacto ambiental" (numeral 8).

En el artículo 9 se declaran los principios ambientales. El principio número 2 indica: "mejor tecnología disponible y mejores prácticas ambientales. El Estado deberá promover en los sectores público y privado, el desarrollo y uso de tecnologías ambientalmente limpias y de energías alternativas no contaminantes y de bajo impacto, que minimicen en todas las fases de una actividad productiva, los riesgos de daños sobre el ambiente, y los costos del tratamiento y 
disposición de sus desechos. Deberá también promover la implementación de mejores prácticas en el diseño, producción, intercambio y consumo sostenible de bienes y servicios, con el fin de evitar o reducir la contaminación y optimizar el uso del recurso natural".

El artículo 24 dispone las "atribuciones de la Autoridad Ambiental Nacional. La Autoridad Ambiental Nacional tendrá las siguientes atribuciones: crear, promover e implementar los incentivos ambientales" (numeral 12).

El artículo 280 declara las "facultades para otorgar incentivos ambientales. La Autoridad Ambiental Nacional, como ente rector, coordinará con otras entidades públicas y privadas el establecimiento de los incentivos ambientales". El artículo 282 establece los "criterios para el otorgamiento de incentivos ambientales: 1. La reducción de los impactos que afectan al ambiente y la prevención de los daños ambientales; 2. El aprovechamiento sostenible de los recursos naturales, la conservación de la biodiversidad y la restauración de los ecosistemas; 3 . La innovación tecnológica y el uso de las mejores técnicas disponibles que causen menos impactos al ambiente; 4. La aplicación de buenas prácticas ambientales y de procesos de producción más limpia; 5. El aprovechamiento racional o eficiente de materiales y de energía; 6 . La reducción o eliminación de materiales tóxicos, emisiones o descargas, y demás medidas que coadyuven en la adaptación y mitigación del cambio climático; 7. La gestión integral de sustancias químicas, residuos y desechos; 8. Los beneficios generados a favor de la población por las medidas o procesos implementados; 9. La capacitación de las personas interesadas para el uso de estos incentivos; y, 10. Los demás que la Autoridad Ambiental Nacional determine”.

El artículo 283 establece los "tipos de incentivos ambientales. Los incentivos ambientales podrán ser: 1. Económicos o no económicos; 2. Fiscales o tributarios; 3. Honoríficos por el buen desempeño ambiental; y, 4. Otros que determine la Autoridad Ambiental Nacional".

\section{Ley de Gestión Ambiental}

Esta Ley establece en su artículo 2, que "la gestión ambiental se sujeta a los principios de solidaridad, corresponsabilidad, cooperación, coordinación, reciclaje y reutilización de desechos, utilización de tecnologías alternativas ambientalmente sustentables y respeto a las culturas y prácticas tradicionales". En su artículo 3 dispone que "el proceso de Gestión Ambiental, se orientará según los principios universales del Desarrollo Sustentable, contenidos en la Declaración de Río de Janeiro de 1992, sobre Medio Ambiente y Desarrollo".

El artículo 8 declara que "la autoridad ambiental nacional será ejercida por el Ministerio del ramo, que actuará como instancia rectora, coordinadora y reguladora del Sistema Nacional Descentralizado de Gestión Ambiental, sin perjuicio de las atribuciones que dentro del ámbito de sus competencias y conforme las leyes que las regulan, ejerzan otras instituciones del Estado".

\section{Las certificaciones ambientales ecuatorianas}

La certificaciones ambientales tienen como propósito ser un camino para las empresas en áreas ambientales, otorgando beneficios desde el punto de vista tanto ecológico como empresarial, motivando a producir e implementar estrategias para cumplir con procesos más 
limpios ayudando en la mejora ambiental y abriendo paso a mercados competitivos (González, 2018).

El Ministerio del Ambiente del Ecuador (MAE) otorga dos certificaciones ambientales, la certificación punto verde y la certificación carbono neutro, con el objetivo de incentivar al sector público y privado, a emplear nuevas y mejores prácticas productivas y de servicios.

La certificación punto verde es una herramienta para fomentar la competitividad del sector industrial y de servicios, comprometiéndolos con la protección y conservación del ambiente. Esta certificación se puede obtiene de dos formas: mediante el proceso de Reconocimiento o Certificación (MAE, 2018).

El Reconocimiento Ecuatoriano Ambiental (REA) Punto Verde se refiere de buenas prácticas ambientales en edificios (entidades públicas y privadas). Para otorgar este reconocimiento se realiza una comparación de indicadores, durante por lo menos dos años de ejecución, de actividades en los ejes temáticos de gestión de desechos, gestión de papel, uso eficiente de agua, energía y combustibles; capacitación y compras responsables (MAE, 2018).

Este reconocimiento se otorga bajo el acuerdo 131 del Ministerio del Ambiente del Ecuador (2012) que tiene "por objeto promover las buenas prácticas en entidades del sector público para apoyar en la reducción de la contaminación ambiental" (artículo 1). En este acuerdo se definen las buenas prácticas ambientales como un compendio de actividades con los que se promueve a diferentes personas jurídicas y naturales a aplicar ciertas prácticas con el fin de reducir la contaminación y los impactos ambientales negativos. Se establece en el artículo 5 la creación del reconocimiento "Reconocimiento Ecuatoriano Ambiental" REA, que "se otorgará a la entidad que reduzca su porcentaje de contaminación a través de buenas prácticas ambientales y frente a las demás entidades públicas". En el artículo 7 señala que "las instituciones implementarán programas de difusión para la disposición adecuada de los desechos, ahorro de agua, ahorro de energía, prohibición de fumar, entre otras disposiciones que sean buenas prácticas ambientales".

La Certificación Ecuatoriana Ambiental (CEA) Punto Verde está dirigida a empresas de producción y servicios que cuente con la Licencia Ambiental correspondiente y demuestren uno o más casos de Producción Limpia. Si la empresa presentara cuatro casos simultáneamente o en un período de hasta 2 años, se le otorgará la máxima certificación como "Empresa EcoEficiente" (MAE, 2018).

El objetivo de la certificación ambiental punto verde es incentivar a las empresas de producción y servicios del Ecuador implementar la estrategia de producción más limpia como una herramienta para el mejoramiento del desempeño ambiental y posicionamiento competitivo de las empresas en el mercado nacional, regional e internacional (MAE, 2018).

Tener una certificación ambiental punto verde favorece la imagen de la empresa otorgando un valor agregado con relación a sus competidores; mediante las certificaciones las empresas se abren paso a nuevos mercados competitivos; se mejora económicamente gracias al control de costos; atrae inversionistas y fuentes de financiamiento, principalmente de aquellos 
con conciencia ambiental; se reducen los impactos ambientales a lo largo del ciclo de vida del producto o servicios; se logran procesos más eficientes con menor uso de materias primas, agua y energía, reduce en cantidad la generación de desechos y emisiones; facilita la gestión de los requisitos legales reduciendo el riesgo de sanciones por la autoridad ambiental, entre otros (MAE, 2018; González, 2018).

El mecanismo para otorgar la certificación ecuatoriana ambiental Punto Verde procesos limpios del Ministerio del Ambiente (2010-2011), expresa son reguladas las empresas de tipo público o privado, que cumplen con actividades productivas y de servicio, las cuales deben someterse al cumplimiento de normativa ambiental vigente, a fin de que en el contexto de su producción puedan verter efluentes líquidos, sólidos o gaseosos de acuerdo a parámetros permitidos. En este contexto, Producción Más Limpia, concebida como una estrategia preventiva ambiental en la que son parte: buenas prácticas ambientales, innovación o cambio de tecnología, debe ser el concepto que se asimile en el marco del desarrollo productivo y de servicio para cumplir con lo estipulado en el marco jurídico nacional e internacional y reducir el riesgo a las personas y al ambiente. Esta certificación tiene beneficios para el ambiente como: uso racional de materias primas, recursos y otros insumos; conservación de los recursos naturales no renovables; reducción de los contaminantes y armonización de las actividades con el ecosistema.

Los ejes temáticos de evaluación que considera el mecanismo son: 1) Materiales: incluye todos aquellos programas enfocados en técnicas de ecodiseño, reducción en el consumo de materias primas y/o residuos, optimización de procesos productivos, reciclaje y manejo de residuos sólidos, sustitución o reducción de materiales y/o sustancias químicas, entre otras opciones aplicadas a las entradas y salidas de los procesos, exceptuando el agua. 2) Agua: incluye todos aquellos programas enfocados a la reducción del consumo de agua, reutilización y/o reciclaje de agua, reducción de efluentes y de la carga contaminante de los efluentes. 3) Energía: incluye todos aquellos programas enfocados en eficiencia energética y energía renovable (uso de fuentes de energía no convencionales, como son: energía eólica, solar, biogas, entre otras). 4) Sistema de Gestión: empresas que cuenten con sistemas que muestren un mejor desempeño ambiental con cualquiera de los recursos antes mencionados.

Otra certificación ecuatoriana que otorga el Ministerio del Ambiente es la certificación carbono neutral, un incentivo que puede obtener el sector público y privado. Esta certificación se otorga a empresas que, como resultado final de una actividad, un proceso o la producción de un bien, la provisión de un servicio o su consumo, no haya emitido más gases efecto invernadero a la atmósfera que los que hayan podido capturar o remover (MAE, 2018).

Dentro de los beneficios de ser carbono neutral se encuentran: mejorar la competitividad e imagen corporativa, al aumentar el valor agregado y preferencia comercial de sus productos y servicios, lo cual posibilita el acceso a nuevos mercados; aumenta la motivación de los empleados y el conocimiento de sus procesos y productos; incorpora el concepto de mejoramiento continuo; contribuye con la mitigación y/o compensación de emisiones; facilita al postulante el acceso a créditos verdes; facilita el acceso a incentivos tributarios para el postulante; logra procesos más eficientes con menor uso de materias primas y energía; reduce la generación de desechos y emisiones e incrementa los beneficios económicos al mejorar el control de los costos (MAE, 2018; González 2018). 
Entre otros beneficios ambientales se mencionan que permite el uso racional de materias primas, recursos y otros insumos; ayuda a conservar los recursos naturales no renovables; reduce emisiones de gases de efecto invernadero; permite una armonía de las actividades productivas con el ecosistema e incentiva la implementación de actividades de conservación (MAE, 2018).

Esta certificación se otorga mediante un Sello de Carbono Neutral y se entrega a las empresas que llegan a un estado de carbono neutro, que es el estado en el que la emisión neta de gases de efecto invernadero (GEI) es cero, es decir, la cantidad de emisiones de GEI equivalen a la cantidad de GEI removidos. Las emisiones de dióxido de carbono pueden ser compensadas de varias maneras: el uso de energías renovables de alta calidad, eficiencia energética y proyectos de reforestación (Ecuador Inmediato, 2011; citado por León, 2015).

Por ello es importante que las empresas logren identificar sus principales fuentes de GEI, de tal manera que estén en capacidad de implementar soluciones reales para la disminución de sus emisiones, lo cual se convierte en el primer paso para alcanzar el sello de carbono neutro y contribuirá a realizar un uso más eficiente de los recursos y se reflejará en la mejora de las finanzas de la empresa.

\section{Las certificaciones ambientales ecuatorianas y su aplicación en las empresas}

Datos aportados por el INEC (2013) de una muestra de 10.646 empresas señalan que $2.734(26 \%)$ empresas tienen algún tipo de certificación ambiental. De esas empresas, el 6\% son certificadas ISO 140001, mientras que el 4\% posee la certificación punto verde (por la gestión de sus desechos, gestión del papel, uso eficiente de agua, energía y combustibles, capacitación y compras responsables) y $1 \%$ la certificación de carbono neutro (compensación de emisiones de dióxido de carbono por el uso de energías renovables de alta calidad, eficiencia energética y proyectos de reforestación) (González, 2018).

León (2015), señala que un gran porcentaje de las empresas ecuatorianas todavía no cuentan con la certificación ambiental Punto Verde, solo el 4\% de las empresas posee este reconocimiento nacional (ver tabla 1). Una de las causas es que las mismas todavía no cuentan con las capacidades para aplicar a esta certificación.

Tabla 1. Empresas con Certificación Punto Verde en Ecuador

\begin{tabular}{cccc}
\hline Tipo de empresa & $\begin{array}{c}\text { \% con certificación } \\
\text { Punto Verde }\end{array}$ & $\begin{array}{c}\text { \% sin certificación } \\
\text { Punto Verde }\end{array}$ & $\begin{array}{c}\text { \% sin Ninguna } \\
\text { Certificación }\end{array}$ \\
\hline Pequeña & 0,8 & 20,4 & 78,8 \\
Mediana & 0,3 & 37,0 & 62,7 \\
Grande & 3,0 & 56,7 & 40,3 \\
\hline
\end{tabular}

Fuente: INEC, 2013

Con relación a la certificación ambiental Carbono Neutro, León (2015) expresa que un elevado porcentaje de empresas no poseen esta certificación, solo el 1\% de las empresas cumplen con los requisitos para alcanzar esta certificación (tabla 2). Esto significa que el $99 \%$ de las empresas no compensa las emisiones de sus actividades económicas, lo cual es negativo para el ambiente.

Tabla 2. Empresas con Certificación Carbono Neutro en Ecuador 


\begin{tabular}{cccc}
\hline Tipo de empresa & $\begin{array}{c}\text { \% con certificación } \\
\text { Punto Verde }\end{array}$ & $\begin{array}{c}\text { \% sin certificación } \\
\text { Punto Verde }\end{array}$ & $\begin{array}{c}\text { \% sin Ninguna } \\
\text { Certificación }\end{array}$ \\
\hline Pequeña & 0,2 & 21,0 & 78,8 \\
Mediana & 0,3 & 37,0 & 62,7 \\
Grande & 0,7 & 59,0 & 40,3 \\
\hline
\end{tabular}

Fuente: INEC, 2013

Las industrias que han recibido el reconocimiento van desde empresas textileras, de alimentos, bebidas, productos de limpieza, siendo Guayas la provincia que posee mayor número de reconocimientos hasta el momento (El Telégrafo, 2017). Entre las empresas que han obtenido la Certificación "Punto Verde" se pueden mencionar: Equisplast, Novacero S. A. - Planta Lasso, Ecuajugos - Nestlé, Cervecería Nacional S. A., Sociedad Agrícola e Industrial San Carlos, Intercia, Contecon, Acería del Ecuador, Corporación Favorita, Cerámicas Rialto (MAE, 2018).

Cervecería Nacional alcanzó la certificación punto verde por registrar la Producción Más Limpia en cuatro Puntos Verdes: 1 . Reducción del 6\% del consumo de agua utilizada, por la reutilización de agua proveniente de la planta de tratamiento de agua residual e incremento de eficiencia en el envasado. 2. Reducción del $4 \%$ en el consumo de energía, por las mejoras en el sistema de cocción y utilización del biogás proveniente de la planta de tratamiento de agua residual. 3. Reducción del 12\% en mermas por la implementación de buenas prácticas en el sistema de embotellado. 4. Reducción del $100 \%$ en el uso de tierras filtrantes por el cambio de tecnología y equipo del proceso de filtración (El Telégrafo, 2017).

El Ministerio de Finanzas fue la primera de las entidades públicas que obtuvo el reconocimiento ambiental punto verde del MAE por cumplir 4 ejes Punto Verde: el primero, la reducción del 4,2\% en consumo de agua; el segundo, la reducción del 11,57\% en consumo de energía, en tercer lugar, creó el programa para control de abastecimiento de combustible en los vehículos de la entidad, y por último, consolidó un Comité Interno para la Gestión de Buenas Prácticas Ambientales en Finanzas (MAE, 2013).

La Secretaría Nacional de la Administración Pública, entidad adscrita al Ejecutivo, que se encarga de mejorar la eficiencia de las instituciones del Estado Central, logró obtener el reconocimiento punto verde por: uso eficiente del agua, disminuyendo su uso a un 15,8\%; ahorro de energía eléctrica por aprovechamiento de la luz natural, bajando así a un 12,6\% el consumo; disminución del 3,7\% en la utilización de gasolina por la optimización y planificación de rutas; cumplimiento en la clasificación, almacenamiento y disposición final de residuos reciclables y desechos peligrosos y especiales; y por realizar capacitaciones constantes al personal en buenas prácticas ambientales (MAE, 2017).

En Ecuador desde el 2012 hasta el 2015, treinta empresas obtuvieron la certificación internacional Carbono Neutro, que se posiciona como un medio para reducir y compensar emisiones que se producen por actividades que realiza cotidianamente el ser humano. Una vez que se tiene cuantificado el inventario de toneladas de $\mathrm{CO} 2$, según la metodología, se reduce un $5 \%$ de esa cantidad y el 95\% restante se lo compensa sembrando árboles que captarán ese porcentaje de contaminación. El Carbono Neutro es el balance entre la cuantificación de las emisiones y las acciones de reducción y remoción/compensación de gases efecto invernadero de una organización en un período verificable. Además, la reducción se obtiene con acciones y 
proyectos de optimización y aprovechamiento de fuentes de energía alternativas de la organización y su entorno. En una empresa la remoción/compensación se logra mediante la protección de bosques o con proyectos específicos que muestren la captura de dióxido de carbono. (El Telégrafo, 2015).

El Ministerio del Ambiente del Ecuador (MAE), con el propósito de liderar las acciones de reducción de emisiones en edificios públicos, emprendió el proyecto Carbono Neutro desde el mes de Mayo de 2010, la experiencia le permitió cuantificar las emisiones de gases de efecto invernadero (GEI) que se generan en su edificio central (Quito) como producto de sus actividades como el consumo energético, el uso de automóviles, transporte de funcionarios, vuelos nacionales e internacionales, generación de residuos. Fue la primera institución pública certificada como carbono neutro. El objetivo es alcanzar "cero emisiones de Gases de Efecto Invernadero" por lo tanto, se establecieron estrategias de reducción como: políticas de uso compartido de vehículos, optimización de rutas de recorrido de trabajadores, separación de residuos, mejora del sistema eléctrico del edificio, menor consumo de agua y electricidad, reutilización de papel, instalación de paneles solares, entre otros, además de planteó un programa de compensación y reducción de emisiones de gases, al reforestar 33 hectáreas con especies nativas en el Área Recreacional El Boliche y así compensar el $95 \%$ de las emisiones generadas (MAE, 2013).

Entre las actividades que realizan las empresas para alcanzar la certificación carbono neutro se encuentran, por ejemplo en el caso del Banco de Guayaquil, primero la eficiencia energética, en donde revisaron sus equipos de aire acondicionado, los cuales ahora cuentan con tecnología para reducir el consumo. Segundo: reducción del consumo de combustibles y aceites, a través de la optimización de rutas de los vehículos y motos de propiedad del banco. Tercero: clasificación de desechos orgánicos e inorgánicos. También se incluyen los tóner y desechos electrónicos, como los generados por los cajeros automáticos. Para gestionar este tipo de desechos, el reciclaje se trabaja con gestores ambientales. Cuarto: movilización de los colaboradores vía aérea. Se analiza que sea la estrictamente necesaria y en su lugar se realizan reuniones a través de videoconferencias, skype y webinar. Además, el Banco Guayaquil apadrina 700 hectáreas del bosque protector El Chamizo-Minas, en el cantón Montufar, provincia de Carchi. Además realiza programas de compensación de C02 a través de la siembra de árboles. Igualmente desarrollaron programa ambiental "Yo Cuido" que se inició en 2014, promoviendo entre sus colaboradores hábitos de conservación y respeto hacia el medio ambiente. Tal iniciativa ha logrado reducir el 5\% de las emisiones de $\mathrm{CO} 2$ de las actividades generadas por el banco (Pinchevsky, 2016).

\section{Las certificaciones ambientales para la competitividad de las empresas}

Heras \& Arana (2011) el hacer la revisión de los resultados obtenidos de investigaciones realizadas por diversos autores sobre la relación gestión ambiental y rendimiento financiero de las empresas, encontraron que los mismos son variados, pero predominan los trabajos en los que se obtiene una relación positiva entre estas dos variables. Los autores mencionados, al analizar la relación existente entre la certificación ISO 14001 y el rendimiento financiero empresarial (medido a través de la rentabilidad económica y el crecimiento de las ventas) con base en un estudio empírico cuantitativo obtuvieron una rentabilidad económica media de las empresas 
certificadas conforme al estándar ISO 14001 superior a la de las empresas no certificadas. En promedio la rentabilidad de las empresas certificadas fue de un 5,91\%, mientras que la rentabilidad de las empresas sin dicho certificado medioambiental fue del 4,32\%. En lo que respecta a la comparativa en los resultados relativos a la variación de ventas, los resultados hallados son también similares, las empresas certificadas tienen en todos los casos un crecimiento de ventas superior al de las empresas no certificadas.

Asimismo, evidenciaron que las empresas certificadas conforme a ISO 14001 eran más rentables que las empresas no certificadas conforme a ISO 14001 tanto en los ejercicios previos como en los ejercicios posteriores a la obtención del certificado. Explican Heras \& Arana (2011) que el rendimiento financiero de las empresas puede influir en su capacidad de abordar iniciativas relacionadas con la gestión medio ambiental, debido a que una empresa con unos buenos resultados financieros puede asignar más recursos a las tecnologías y actividades dirigidas a la implantación de actuaciones en materia medio ambiental, como la certificación de sistema de gestión medioambiental.

La investigación realizada por Acuña, Figueroa \& Wilches (2017) sobre un grupo de empresas manufactureras de Barranquilla con certificación ISO 14001, identificaron los beneficios de la implementación de este sistema administrativo, orientado por la planeación estratégica de la organización en la rentabilidad de la empresa, como lo son una mejor imagen corporativa, un mejor desempeño jurídico, un adecuado manejo de los recursos, la reducción en la generación de residuos y la atracción de nuevos clientes; ratificándose este como una herramienta importante de la administración actual y vital para la generación de ventajas competitivas.

Los autores Acuña, Figueroa \& Wilches (2017) destacan que los sistemas de gestión y las certificaciones se han convertido en una parte imprescindible en la organización y en un requisito para la supervivencia en el siglo XXI, pero no todas las organizaciones han alcanzado el mismo nivel de madurez en sistemas de gestión ambiental, algunas contemplan la gestión ambiental como un instrumento para mejorar la eficiencia de sus procesos, al mismo tiempo que cuidan el medio ambiente y muchas otras se limitan a cumplir los requisitos de la legislación. En efecto, las pequeñas y medianas empresas que no buscan colocar sus productos en mercados externos, optan por implantar un sistema de gestión ambiental que les permita cumplir con la normativa legal vigente en sus países de operación. Las empresas grandes, por el contrario, suelen requerir la certificación ISO 14001 para competir exitosamente en mercados más exigentes.

Bermeo \& Saavedra (2018) en su trabajo investigación sobre la competitividad sistémica de la mipyme manufacturera en el nivel micro: caso de la fabricación de muebles de madera en Ecuador, encontraron que el nivel de competitividad en el área de gestión ambiental es el pico que mayormente sobresale, sin embargo y a pesar que refleja un porcentaje medianamente importante por acercarse casi la mitad del total de las empresas, no existen directrices claras sobre sobre lo que realmente implica un manejo ambiental adecuado en este sector de la producción. Ciertas buenas prácticas básicas hacen que el empresario sienta que cumple con esta obligación, sin embargo muchas veces las realiza para cumplir con las regulaciones a las que se encuentra sujeta la industria, dado que la mayoría de ellos considera que implica costos elevados, además, la gestión ambiental la relacionan con el aseguramiento de la calidad. 
El estudio demostró que el aseguramiento de la calidad se relaciona directamente con la gestión ambiental e impactan en la competitividad de la mipyme manufacturera. Los estándares fijados en el tema ambiental son cada vez más altos e incluso las buenas prácticas que las empresas realizan son evaluadas por los organismos de control público. En la ciudad de Cuenca, el tema medioambiente está tomando fuerza, y el hallazgo probado es que la gestión ambiental de las empresas se encuentra relacionado directamente con el aseguramiento de la calidad que tenga la misma, pues los parámetros de producción observando los requisitos de calidad exigen que se cumplan los lineamientos de gestión ambiental (Bermeo \& Saavedra, 2018).

En este sentido, señala González (2017), la gestión ambiental y la competitividad poseen relación directa, por ello la necesidad de implementar sistemas de gestión ambiental en las pymes donde la alta dirección asuma su compromiso, además es necesario la participación total de los trabajadores para hacer frente y manejar los problemas ambientales así como plantear los objetivos ambientales; el entrenamiento y la formación de habilidades en los trabajadores para cumplir sus responsabilidades ambientales y lograr objetivos ambientales, y por último el uso de indicadores ambientales para medir el desempeño ambiental de las empresas, de tal manera que se minimice el impacto negativo sobre el ambiente.

Por su parte, Bravo \& Martínez (2016) en su trabajo sobre eficiencia energética y competitividad empresarial y economía verde expresan que la energía cada día se encarece más, por ello en muchos casos una de las principales partidas del costo total es el costo energético, donde se incluyen los componentes relativos a la producción, distribución y uso de las diferentes formas de energía y el agua, de allí que sea necesaria la eficiencia energética, entendida como la eficiencia en la producción, distribución y uso de la energía, necesaria para garantizar la calidad total. Los aspectos básicos que determinan la competitividad de una empresa o institución son la calidad y el precio de sus productos o servicios. La posición en el mercado y la estrategia de cambio de posición vienen determinadas por la relación calidad-precio con respecto a otras empresas de la competencia.

\section{Conclusión}

Las certificaciones ambientales ecuatorianas tienen basamento en la legislación del país, siendo el Ministerio del Ambiente la autoridad ambiental y el ente encargado de su promoción e implementación con el fin de mejorar su desempeño ambiental.

Las certificaciones ambientales que otorga en Ministerio del Ambiente del Ecuador son la certificación punto verde y la certificación carbono neutro, las cuales tienen como objetivo incentivar el empleo de prácticas productivas y de servicios con el fin de reducir la contaminación y los impactos ambientales negativos e implementar una producción más limpia como una herramienta para el mejoramiento del desempeño ambiental y posicionamiento competitivo de las empresas en el mercado nacional, regional e internacional.

Entre las ventajas de las certificaciones ambientales en las empresas destacan: favorece la imagen de la empresa, la entrada a nuevos mercados competitivos; se mejora económicamente gracias al control de costos; atrae inversionistas y fuentes de financiamiento, principalmente de aquellos con conciencia ambiental; se reducen los impactos ambientales a lo largo del ciclo de 
vida del producto o servicios; se logran procesos más eficientes con menor uso de materias primas, agua y energía, reduce en cantidad la generación de desechos y emisiones; facilita la gestión de los requisitos legales reduciendo el riesgo de sanciones por la autoridad ambiental.

Un gran porcentaje de las empresas ecuatorianas todavía no cuentan con la certificación ambiental Punto Verde y Carbono Neutro, siendo una de las causas que las mismas todavía no cuentan con las capacidades para aplicar a esta certificación. Las industrias que han recibido el reconocimiento van desde empresas textileras, alimentos, bebidas, productos de limpieza, siendo Guayas la provincia que mayor número de reconocimientos ha alcanzado hasta el momento.

Las certificaciones ambientales contribuyen a que las empresas mejoren su competitividad a través de la rentabilidad económica y el crecimiento de las ventas, además de una mejor imagen corporativa, un mejor desempeño legal, un adecuado manejo de los recursos, la reducción en la generación de residuos y la atracción de nuevos clientes.

\section{Bibliografía}

Acuerdo 131 del Ministerio del Ambiente del Ecuador (2012). Recuperado de: http://www.ambiente.gob.ec/wp-content/uploads/downloads/2012/10/Acuerdo-Ministerial131.pdf

Acuña, N.; Figueroa, L. \& Wilches, M. J. (2017). Influencia de los Sistemas de Gestión Ambiental ISO 14001 en las organizaciones: caso estudio empresas manufactureras de Barranquilla. Ingeniare. Revista chilena de ingeniería, 25 (1), 143-153. Recuperado de: www.redalyc.org/pdf/772/77249637013.pdf

Bermeo Pazmiño, K. V. \& Saavedra García, M. L. (2018). La competitividad sistémica de la mipyme manufacturera en el nivel micro: caso de la fabricación de muebles de madera, Ecuador. Small Business International Review, 2 (1), 1-15. Recuperado de: https://doi.org/10.26784/sbir.v2i1.20

Bravo Hidalgo, D. \& Martínez Pérez, Y. (2016). Eficiencia energética, competitividad empresarial y economía verde. Revista Publicando, 3(9).2016, 447-466. Recuperado de: https://dialnet.unirioja.es/descarga/articulo/5833551.pdf

Código Orgánico del Ambiente. (2017). Registro Oficial Suplemento 983 de 12-abr.-2017. Recuperado de http://www.ambiente.gob.ec/wpcontent/uploads/downloads/2018/01/CODIGO_ORGANICO_AMBIENTE.pdf

Constitución de la República del Ecuador. (2008). Recuperado de: http://www.inocar.mil.ec/web/images/lotaip/2015/literal_a/base_legal/A._Constitucion_republica _ecuador_2008constitucion.pdf

El Telégrafo. (10 de mayo de 2017). Ministerio del Ambiente entregó 130 certificados 'Punto Verde' en menos de 5 años. Recuperado de:

https://www.eltelegrafo.com.ec/noticias/sociedad/6/ministerio-del-ambiente-entrego-130certificados-punto-verde-en-menos-de-5-anos

El Telégrafo. (22 de marzo de 2015). 30 empresas obtienen certificado ambiental. Recuperado de: https://www.eltelegrafo.com.ec/noticias/economia/8/30-empresas-obtienen-certificado-ambiental 
González Ordóñez, A. I. (2017). La gestión ambiental en la competitividad de las Pymes. Revista científica Agroecosistemas, 5 (1), 60-70. Recuperado de http://aes.ucf.edu.cu/index.php/aes/index

González, A. (2018). Prácticas ambientales en Pymes ecuatorianas. Revista Arjé, 12 (22), Edición Especial Enero-Julio 2018. Recuperado de: www.arje.bc.uc.edu.ve/arje22e/art04.pdf

Heras Saizarbitoria, I. \& Arana Landin, G. (2011). Impacto de la certificación ISO 14001 en el rendimiento financiero empresarial: conclusiones de un estudio empírico. Cuadernos de Economía y Dirección de la Empresa, 14, 112-122. Recuperado de: https://www.sciencedirect.com/science/article/pii/S1138575811000089

Instituto Nacional de Estadística y Censos. (2013). Encuesta de Información Ambiental Económica en Empresas. Recuperado de: www.ecuadorencifras.gob.ve.

León Bolaños, N. V. (2015). Propuesta de instrumentos de política pública que promuevan la protección y cuidado ambiental en la gestión empresarial del Ecuador. Tesis de grado para la obtención del grado de Economista. Pontificia Universidad Católica del Ecuador, 135. Recuperado de: http://repositorio.puce.edu.ec/bitstream/handle/220

00/10481/Disertaci\%C3\%B3n\%20completa.pdf?sequence=1\&isAllowed=y.

Ley de Gestión Ambiental. (2004). Registro Oficial Suplemento 418. Recuperado de: www.ambiente.gob.ec/wp-content/uploads/.../ley-de-gestión-ambiental.pdf.

MAE (Ministerio del Ambiente del Ecuador). 2013. Recuperado de: http://www.ambiente.gob.ec/un-puntoverde-al-ministerio-de-finanzas-el-primero-para-entidades-publicas/ www.ambiente.gob.ec/el-28de-enero-se-celebro-el-dia-mundial-por-la-reduccion-de-las-emisiones-de-co2-dioxido-decarbono/

MAE (Ministerio del Ambiente del Ecuador). 2017. Recuperado de: http://www.ambiente.gob.ec/lasecretaria-nacional-de-la-administracion-vpublica-y-la-cerveceria-nacional-s-a-recibieron-lacertificacion-ambiental-punto-verde/

MAE (Ministerio del Ambiente del Ecuador). 2018. Recuperado de: http://www.ambiente.gob.ec/puntoverde/ http://www.ambiente.gob.ec/mae-presento-oficialmente-el-reconocimiento-ecuatorianoambiental-carbono-neutral/

Mecanismo para otorgar la certificación ecuatoriana ambiental Punto Verde procesos limpios del Ministerio del Ambiente (2010-2011). Recuperado de http://www.ambiente.gob.ec/wpcontent/uploads/downloads/2013/12/Mecanismo-Certificacion-AM225-Punto-Verde.pdf

Pinchevsky, M. (2016). Empresas reducen su huella de carbono. Revista El Universo Ecuador. 27 de marzo de 2016. Recuperado de http://www.larevista.ec/actualidad/ecologia/empresas-reducen-su-huellade-carbono

Rodríguez Becerra, M., \& Van Hoof, B. (2004). Para que la Pyme sea más competitiva se requiere una gestión ambiental preventiva. Recuperado de www.manuelrodriguezbecerra.org/bajar/Pymes.pd 